\title{
A Discrete Ortholithiated Acetophenone Imine Derivative: Isolation, Characterization, and Synthesis of Group IV Metal Complexes
}

\author{
Tamam I. Baiz and Joseph A. R. Schmidt* \\ Department of Chemistry, The University of Toledo, 2801 W. Bancroft St. MS 602 \\ Toledo, Ohio 43606-3390 \\ Joseph.Schmidt@utoledo.edu
}

\section{Supporting Information}

Experimental procedures and characterization data for 1-7. Experimental conditions and detailed values for the $\mathrm{K}_{\mathrm{eq}}$ of $\mathbf{3}$. Crystallographic procedures and crystal data for 4 (10 pages). 


\section{Experimental Section}

General Considerations. Compound 1 was synthesized under ambient atmosphere, whereas all other reactions were performed under standard Schlenk and glovebox techniques. All glassware was dried overnight and purged three times using vacuum-nitrogen filling cycles before use. All cannulae were oven-dried and quickly transferred to the reaction flask to be flushed under nitrogen once cooled to room temperature. Pentane and toluene were passed through columns of activated molecular sieves and degassed with nitrogen. Ether was passed through a column of activated alumina and degassed with nitrogen. $\mathrm{ZrCl}_{4}, \mathrm{TiCl}_{4}, 3$ ',4'-methylenedioxyacetophenone, $p$ toluenesulfonic acid, 2,6-diethylaniline, and ${ }^{\mathrm{n}} \mathrm{BuLi}$ were used as purchased. Trimethylsilylmethyllithium was purchased as a solution in pentane and used as a solid after evaporation of the solvent. Piperonal was left under vacuum overnight to remove any moisture. $(\text { py })_{2} \mathrm{Ti}\left(=\mathrm{N}^{t} \mathrm{Bu}\right) \mathrm{Cl}_{2}$ was synthesized as previously reported. ${ }^{1} \mathrm{C}_{6} \mathrm{D}_{6}$ was first degassed by several freeze-pump-thaw cycles and then vacuum transferred from $\mathrm{Na}$ /benzophenone. ${ }^{1} \mathrm{H},{ }^{13} \mathrm{C}\left\{{ }^{1} \mathrm{H}\right\}$ and ${ }^{7} \mathrm{Li}\left\{{ }^{1} \mathrm{H}\right\}$ NMR samples were prepared in the glovebox under nitrogen and the spectra were recorded in $\mathrm{C}_{6} \mathrm{D}_{6}$ at ambient temperatures on a VXRS 400 or Inova $600 \mathrm{MHz}$ spectrometer. ${ }^{1} \mathrm{H}$ NMR chemical shifts are given relative to $\mathrm{C}_{6} \mathrm{D}_{5} \mathrm{H}(\delta 7.16),{ }^{7} \mathrm{Li}$ NMR spectra are relative to an external $\mathrm{LiCl}(3.00 \mathrm{M}$ in $\left.\mathrm{D}_{2} \mathrm{O}\right)$ standard $(\delta 0.00)$ and ${ }^{13} \mathrm{C}$ NMR chemical shifts are relative to $\mathrm{C}_{6} \mathrm{D}_{6}(\delta 128.0)$. Melting points were acquired in sealed capillary tubes under nitrogen and are uncorrected. IR samples were prepared as Nujol mulls. Single crystal X-ray structure determination was performed at The University of Toledo. Elemental analyses were performed by Desert Analytics, Tucson, AZ. 
L-H (1). 2,6-Diethylaniline (12 g, $79 \mathrm{mmol}$ ) was added to a solution of 3',4'methylenedioxyacetophenone $(10 \mathrm{~g}, 61 \mathrm{mmol})$ in toluene $(250 \mathrm{~mL}) \cdot p$-Toluenesulfonic acid $(2.0 \mathrm{~g})$ was added, and the resulting solution was left to reflux for $2 \mathrm{~d}$, after which the flask was cooled to room temperature. After removal of volatiles under vacuum, the resulting oil was dissolved in $\mathrm{CH}_{2} \mathrm{Cl}_{2}(150 \mathrm{~mL})$. The solution obtained was neutralized with saturated $\mathrm{NaHCO}_{3(\mathrm{aq})}$. The organic layer was collected and washed with distilled water $(75 \mathrm{~mL})$ and dried over anhydrous $\mathrm{MgSO}_{4}$. The solution was filtered and the solvent evaporated. Vacuum distillation gave a light brown oil that solidified after cooling to room temperature; (15 g, 85\%); mp $43-44{ }^{\circ} \mathrm{C} ;{ }^{1} \mathrm{H}$ NMR $\left(\mathrm{C}_{6} \mathrm{D}_{6}, 400 \mathrm{MHz}\right): \delta$ $7.90(\mathrm{~d}, 1 \mathrm{H}, 2 \mathrm{~Hz}), 7.26$ (dd, $\left.1 \mathrm{H},{ }^{3} J=8 \mathrm{~Hz},{ }^{4} J=2 \mathrm{~Hz}\right), 7.12-7.03$ (m, 3H), 6.64 (d, $1 \mathrm{H}, 8$ $\mathrm{Hz}), 5.28(\mathrm{~s}, 2 \mathrm{H}), 2.47-2.28(\mathrm{~m}, 4 \mathrm{H}), 1.72(\mathrm{~s}, 3 \mathrm{H}), 1.11(\mathrm{t}, 6 \mathrm{H}, 8 \mathrm{~Hz}) ;{ }^{13} \mathrm{C}\left\{{ }^{1} \mathrm{H}\right\} \mathrm{NMR}$ $\left(\mathrm{C}_{6} \mathrm{D}_{6}, 100 \mathrm{MHz}\right): \delta 163.17,150.07,148.71,148.55,134.03,131.74,126.41,123.37$, 122.30, 107.76, 107.52, 101.38, 25.21, 17.28, 14.02; IR: 2952 (s), 2924 (s), 2854 (s), $2726(w), 1636(w), 1607(w), 1591(w), 1505(m), 1487(m), 1464$ (s), 1450 (s), 1377 (s), 1350 (s), 1343 (m), 1289 (m), 1256 (s), 1222 (w), $1195(w), 1154(w), 1112(w)$, $1107(w), 1043(\mathrm{~m}), 951(w), 943(w), 899(w), 886(w), 857(w), 824(w), 805(w), 770$ (w), $722(\mathrm{~m}), 631$ (w). Anal. Calcd for $\mathrm{C}_{19} \mathrm{H}_{21} \mathrm{NO}_{2}: \mathrm{C}, 77.26$; H, 7.17; N, 4.74. Found: $\mathrm{C}$, 77.17; H, 7.33: N, 4.60 .

L-Li (2). A solution of ${ }^{\mathrm{n}} \mathrm{BuLi}(1.51 \mathrm{M}$ in hexane, $22 \mathrm{~mL}, 34 \mathrm{mmol})$ was slowly added via syringe to a solution of $\mathbf{1}(10 \mathrm{~g}, 34 \mathrm{mmol})$ in pentane $(200 \mathrm{~mL})$ at $0{ }^{\circ} \mathrm{C}$. The resulting solution was left to stir at $0{ }^{\circ} \mathrm{C}$ for $30 \mathrm{~min}$ and then warmed to room temperature for an additional $3 \mathrm{~h}$. The mixture was filtered and the orange precipitate washed with pentane $(2 \times 80 \mathrm{~mL})$. Drying under vacuum yielded an orange solid; $(9.2 \mathrm{~g}, 90 \%)$; $\mathrm{mp}$ 
$129{ }^{\circ} \mathrm{C}(\mathrm{dec}.) ;{ }^{1} \mathrm{H}$ NMR $\left(\mathrm{C}_{6} \mathrm{D}_{6}, 400 \mathrm{MHz}\right): \delta 7.21$ (d, 1H, 8Hz), 6.94 (s, 3H), $6.66(\mathrm{~d}, 1 \mathrm{H}$, $8 \mathrm{~Hz}), 4.81(\mathrm{~s}, 2 \mathrm{H}), 2.22(\mathrm{~m}, 4 \mathrm{H}), 1.85(\mathrm{~s}, 3 \mathrm{H}), 0.92(\mathrm{t}, 6 \mathrm{H}, 8 \mathrm{~Hz}) ;{ }^{13} \mathrm{C}\left\{{ }^{1} \mathrm{H}\right\} \mathrm{NMR}\left(\mathrm{C}_{6} \mathrm{D}_{6}\right.$, $100 \mathrm{MHz}): \delta 177.0,155.9,147.9,144.2,143.7,133.5,126.3,124.9,124.0,104.9,97.6$, 24.8, 17.8, 14.1; ${ }^{7} \mathrm{Li}\left\{{ }^{1} \mathrm{H}\right\}$ NMR $\left(\mathrm{C}_{6} \mathrm{D}_{6}, 155 \mathrm{MHz}\right): \delta$ 4.08; IR: 2916 (s), 2854 (s), 2950 (s), $2726(\mathrm{w}), 1615(\mathrm{~m}), 1589(\mathrm{w}), 1558(\mathrm{~m}), 1487$ (m), $1460(\mathrm{~s}), 1459$ (s), $1378(\mathrm{~s}), 1322$ (m), $1279(\mathrm{~m}), 1256(\mathrm{~m}), 1242(\mathrm{~m}), 1194$ (w), $1130(\mathrm{w}), 1110(\mathrm{w}), 1093(\mathrm{w}), 1082(\mathrm{w})$, $1038(\mathrm{~m}), 981(\mathrm{w}), 927(\mathrm{~m}), 874(\mathrm{w}), 839(\mathrm{w}), 825(\mathrm{w}), 804(\mathrm{w}), 794(\mathrm{w}), 786(\mathrm{w}), 775$ (m), 722 (w), 643 (w). Anal. Calcd for $\mathrm{C}_{19} \mathrm{H}_{20} \mathrm{NO}_{2} \mathrm{Li}$ : C, 75.73; H, 6.69; N, 4.64. Found: C, 75.61; H, 6.89; N, 4.75.

$\mathbf{L}_{2} \mathbf{Z r C l}_{\mathbf{2}}$ (3). The lithiated ligand $\mathbf{2}(4.0 \mathrm{~g}, 13 \mathrm{mmol})$ and $\mathrm{ZrCl}_{4}(1.5 \mathrm{~g}, 6.4 \mathrm{mmol})$ were placed in a $250 \mathrm{~mL}$ flask. Toluene $(180 \mathrm{~mL})$ at $0{ }^{\circ} \mathrm{C}$ was added. The resulting dark red solution was left to stir at $0{ }^{\circ} \mathrm{C}$ for $30 \mathrm{~min}$, after which it was removed from the ice bath and allowed to warm to room temperature. The solution was then stirred at room temperature overnight. The solution was filtered and half of the solvent was evaporated. Pentane $(70 \mathrm{~mL})$ was added and the solution was then placed in the freezer at $-25{ }^{\circ} \mathrm{C}$ overnight. After filtration, the remaining orange solid was washed with pentane $(50 \mathrm{~mL})$ and dried; (4.1 g, 85\%); mp $144{ }^{\circ} \mathrm{C}\left(\mathrm{dec}\right.$.); ${ }^{1} \mathrm{H}$ NMR $\left(\mathrm{C}_{6} \mathrm{D}_{6}, 400 \mathrm{MHz}\right)$ : Complex 3a: $\delta 7.04$ (br s, 6H), $6.76(\mathrm{~d}, 2 \mathrm{H}, 8 \mathrm{~Hz}), 6.42(\mathrm{~d}, 2 \mathrm{H}, 8 \mathrm{~Hz}), 5.39$ (d, 2H, $2 \mathrm{~Hz}), 5.10(\mathrm{~d}, 2 \mathrm{H}$, $2 \mathrm{~Hz}), 3.10\left(\mathrm{dq}, 2 \mathrm{H},{ }^{2} J=16 \mathrm{~Hz},{ }^{3} J=8 \mathrm{~Hz}\right), 2.97\left(\mathrm{dq}, 2 \mathrm{H},{ }^{2} J=16 \mathrm{~Hz},{ }^{3} J=8 \mathrm{~Hz}\right), 2.85$ (dq, $2 \mathrm{H},{ }^{2} J=16 \mathrm{~Hz},{ }^{3} J=8 \mathrm{~Hz}$ ), $2.55\left(\mathrm{dq}, 2 \mathrm{H},{ }^{2} J=16 \mathrm{~Hz},{ }^{3} J=8 \mathrm{~Hz}\right), 1.79$ (s, 6H), 1.20 (t, 6H, $8 \mathrm{~Hz}), 1.12$ (t, 6H, $8 \mathrm{~Hz}$ ); Complex 3b: $\delta 7.05$ (br s, 6H), 6.69 (d, 2H, $8 \mathrm{~Hz}), 6.35$ (d, $2 \mathrm{H}, 8 \mathrm{~Hz}), 5.16(\mathrm{~d}, 2 \mathrm{H}, 2 \mathrm{~Hz}), 5.01(\mathrm{~d}, 2 \mathrm{H}, 2 \mathrm{~Hz}), 3.30\left(\mathrm{dq}, 2 \mathrm{H},{ }^{2} J=16 \mathrm{~Hz},{ }^{3} J=8 \mathrm{~Hz}\right)$, 2.44-2.20 (m, 6H), 1.78 (s, 6H), 1.27 (t, 6H, $8 \mathrm{~Hz}), 0.76(\mathrm{t}, 6 \mathrm{H}, 8 \mathrm{~Hz}) ;{ }^{13} \mathrm{C}\left\{{ }^{1} \mathrm{H}\right\} \mathrm{NMR}$ 
$\left(\mathrm{C}_{6} \mathrm{D}_{6}, 100 \mathrm{MHz}\right): \delta 188.6,185.7,167.9,164.4,150.6,149.8,145.4,139.6,138.9,137.1$, $136.3,135.6,127.4,126.5,126.4,126.3,126.0,125.9,125.8,124.6,123.7,123.3,106.7$, $106.5,101.3,100.6,100.2,26.1,25.3,25.2,24.5,24.3,18.5,18.4,14.6,14.4,14.3,14.0$ IR: 2954 (s), 2923 (s), 2853 (s), 1572 (w), 1539 (w), 1497 (w), 1462 (s), 1411 (w), 1377 (m), $1353(w), 1309(w), 1248(w), 1181(w), 1155(w), 1148(w), 1122(w), 1095(w)$, $1051(w), 946(w), 934(w), 861(w), 812(w), 802(w), 785(w), 779(w), 722(w)$.

$\mathbf{L}_{2} \mathbf{Z r}\left(\mathbf{C H}_{2} \mathrm{SiMe}_{3}\right)_{2}$ (4). $\mathrm{LiCH}_{2} \mathrm{SiMe}_{3}(75 \mathrm{mg}, 0.79 \mathrm{mmol})$ in toluene $(10 \mathrm{~mL})$ was added via cannula to a slurry of $\mathbf{3}(300 \mathrm{mg}, 0.40 \mathrm{mmol})$ in toluene $(30 \mathrm{~mL})$. The reddishorange solution was left to stir at room temperature overnight. The solvent was evaporated to give a solid. Pentane $(30 \mathrm{~mL})$ was added and then evaporated in an effort to remove residual toluene. Ether $(30 \mathrm{~mL})$ was then added and the solution was filtered twice. After concentrating down to half its volume, cooling the solution to $-25{ }^{\circ} \mathrm{C}$ overnight resulted in yellow crystals; $(150 \mathrm{mg}, 45 \%) ;{ }^{1} \mathrm{H}$ NMR $\left(\mathrm{C}_{6} \mathrm{D}_{6}, 400 \mathrm{MHz}\right): \delta 7.11$ (d, 2H, $7 \mathrm{~Hz}), 6.94(\mathrm{t}, 2 \mathrm{H}, 7 \mathrm{~Hz}), 6.75(\mathrm{~d}, 2 \mathrm{H}, 8 \mathrm{~Hz}), 6.67$ (d, 2H, $7 \mathrm{~Hz}), 6.46$ (d, 2H, 8 Hz), 5.32 (d, 2H, $2 \mathrm{~Hz}), 4.96$ (d, 2H, $2 \mathrm{~Hz}), 2.96\left(\mathrm{dq}, 2 \mathrm{H},{ }^{2} J=16 \mathrm{~Hz},{ }^{3} J=8 \mathrm{~Hz}\right), 2.48$ $\left(\mathrm{dq}, 2 \mathrm{H},{ }^{2} J=16 \mathrm{~Hz},{ }^{3} J=8 \mathrm{~Hz}\right), 2.08\left(\mathrm{dq}, 2 \mathrm{H},{ }^{2} J=16 \mathrm{~Hz},{ }^{3} J=8 \mathrm{~Hz}\right), 1.92\left(\mathrm{dq}, 2 \mathrm{H},{ }^{2} J=\right.$ $\left.16 \mathrm{~Hz},{ }^{3} J=8 \mathrm{~Hz}\right), 1.84(\mathrm{~d}, 2 \mathrm{H}, 11 \mathrm{~Hz}), 1.73(\mathrm{~s}, 6 \mathrm{H}), 1.70(\mathrm{~d}, 2 \mathrm{H}, 11 \mathrm{~Hz}), 1.28(\mathrm{t}, 6 \mathrm{H}, 8$ $\mathrm{Hz}), 0.72(\mathrm{t}, 6 \mathrm{H}, 8 \mathrm{~Hz}), 0.22(\mathrm{~s}, 18 \mathrm{H}) ;{ }^{13} \mathrm{C}\left\{{ }^{1} \mathrm{H}\right\}$ NMR $\left(\mathrm{C}_{6} \mathrm{D}_{6}, 100 \mathrm{MHz}\right): \delta$ 182.1, 165.9, $151.9,149.5,146.2,140.7,136.0,135.0,125.7,125.2,124.8,123.4,106.3,100.1,73.5$, $25.9,24.5,18.8,14.5,14.4,3.7$.

$\mathbf{L}_{2} \mathbf{T i C l}_{\mathbf{2}}$ (5). A solution of $\mathrm{TiCl}_{4}(1.7 \mathrm{~g}, 9.0 \mathrm{mmol})$ in toluene $(10 \mathrm{~mL})$ at $0{ }^{\circ} \mathrm{C}$ was added to a solution of $2(5.4 \mathrm{~g}, 18 \mathrm{mmol})$ in toluene $(70 \mathrm{~mL})$ at $0{ }^{\circ} \mathrm{C}$. The resulting solution was left to react at $0{ }^{\circ} \mathrm{C}$ for $2 \mathrm{~h}$, after which it was allowed to warm to room 
temperature. After an additional $2 \mathrm{~h}$, the solution was filtered and the solvent was evaporated. The solid obtained was washed with pentane $(50 \mathrm{~mL})$ and dried under vacuum; (5.4 g, 85\%); mp $>200{ }^{\circ} \mathrm{C} ;{ }^{1} \mathrm{H}$ NMR $\left(\mathrm{C}_{6} \mathrm{D}_{6}, 400 \mathrm{MHz}\right): \delta$ 7.12-6.98 (m, 8H), $6.66(\mathrm{~d}, 2 \mathrm{H}, 8 \mathrm{~Hz}), 5.37$ (s, 2H), $5.22(\mathrm{~s}, 2 \mathrm{H}), 2.39-2.16(\mathrm{~m}, 8 \mathrm{H}), 1.86(\mathrm{~s}, 6 \mathrm{H}), 1.09-1.05$ (m, 12H); ${ }^{13} \mathrm{C}\left\{{ }^{1} \mathrm{H}\right\}$ NMR $\left(\mathrm{C}_{6} \mathrm{D}_{6}, 100 \mathrm{MHz}\right): \delta 167.3,148.6,148.1,146.8,131.8,131.5$, $127.4,126.4,126.0,123.4,122.5,116.4,107.7,101.3,25.2,24.3,17.3,14.2,14.0$; IR: 2946 (s), 2917 (s), 2858 (s), 2726 (w), 1634 (w), 1590 (w), 1540 (w), 1461 (s), 1377 (m), $1341(w), 1306$ (w), 1289 (w), 1247 (m), 1189 (w), 1127 (w), $1110(w), 1090(w), 1050$ (w), $1038(w), 938(w), 910(w), 843(w), 805(w), 776(w), 722(w)$.

$\mathbf{L}_{2} \mathbf{T i}=\mathbf{N}^{t} \mathbf{B u}(\mathbf{6})$. A solution of $\mathbf{2}(2.0 \mathrm{~g}, 6.6 \mathrm{mmol})$ in toluene $(50 \mathrm{~mL})$ was added to a solution of $\mathrm{Ti}\left(=\mathrm{N}^{\mathrm{t}} \mathrm{Bu}\right) \mathrm{Cl}_{2}(\mathrm{py})_{2}(1.2 \mathrm{~g}, 3.3 \mathrm{mmol})$ in toluene $(30 \mathrm{~mL})$ at $0{ }^{\circ} \mathrm{C}$. The resulting solution was left to react at $0{ }^{\circ} \mathrm{C}$ for $30 \mathrm{~min}$, after which it was allowed to warm to room temperature, where it was left to react for $2.5 \mathrm{~h}$. The solution was then filtered and the solvent was evaporated to give a dark red solid. The product was recrystallized from pentane; (1.9 g, 81\%); mp $183{ }^{\circ} \mathrm{C}(\mathrm{dec}) ;{ }^{1} \mathrm{H}$ NMR $\left(\mathrm{C}_{6} \mathrm{D}_{6}, 400 \mathrm{MHz}\right): \delta$ 7.06-6.98 (m, 4H), $6.86(\mathrm{~d}, 2 \mathrm{H}, 8 \mathrm{~Hz}), 6.77$ (d, 2H, $8 \mathrm{~Hz}), 6.49$ (d, 2H, $8 \mathrm{~Hz}), 5.48$ (d, 2H, $2 \mathrm{~Hz})$, $5.02(\mathrm{~d}, 2 \mathrm{H}, 2 \mathrm{~Hz}), 3.30(\mathrm{~m}, 2 \mathrm{H}), 2.70(\mathrm{~m}, 2 \mathrm{H}), 1.90(\mathrm{~m}, 4 \mathrm{H}), 1.64$ (s, 6H), 1.36 (s, 9H), $1.29(\mathrm{t}, 6 \mathrm{H}, 8 \mathrm{~Hz}), 0.72(\mathrm{t}, 6 \mathrm{H}, 8 \mathrm{~Hz}) ;{ }^{13} \mathrm{C}\left\{{ }^{1} \mathrm{H}\right\} \mathrm{NMR}\left(\mathrm{C}_{6} \mathrm{D}_{6}, 100 \mathrm{MHz}\right): \delta$ 185.8, 167.7, $153.3,148.2,145.5,138.0,137.5,134.8,125.6,124.6,124.4,123.2,104.9,99.7,71.7$, 33.2, 24.5, 24.4, 17.5, 13.1, 12.9; IR: 2959 (s), 2915 (s), 2857 (s), 2724 (w), 1599 (w), $1540(\mathrm{~m}), 1462$ (s), 1406 (m), 1377 (s), 1348 (w), $1304(\mathrm{~m}), 1250(\mathrm{~m}), 1206(\mathrm{w}), 1187$ (w), $1142($ (w), $1114(w), 1091(w), 1049(w), 988$ (w), $942(w), 932(w), 896(w), 863$ 
(w), 807 (w), 794 (w), 780 (w), 722 (w). Anal. Calcd for $\mathrm{C}_{42} \mathrm{H}_{49} \mathrm{~N}_{3} \mathrm{O}_{4} \mathrm{Ti} \cdot \mathrm{LiCl}$ : C, 69.21; H, 6.78; N, 5.76. Found: C, 68.71; H, 6.38; N, 6.14.

NMR Scale Synthesis of $\mathbf{L}_{\mathbf{2}} \mathbf{T i}=\mathbf{O}$ (7). Piperonal (3.2 $\mathrm{mg}, 0.021 \mathrm{mmol}$ ) was added to a solution of $6(15 \mathrm{mg}, 0.021 \mathrm{mmol})$ in $\mathrm{C}_{6} \mathrm{D}_{6}(1.0 \mathrm{~mL})$. In addition to the generation of byproduct piperonal tert-butyl imine, 7 was immediately observed by ${ }^{1} \mathrm{H}$ NMR spectroscopy: ${ }^{1} \mathrm{H}$ NMR $\left(\mathrm{C}_{6} \mathrm{D}_{6}, 400 \mathrm{MHz}\right): \delta$ 7.12-6.96 (m, 6H), $6.62(\mathrm{~d}, 2 \mathrm{H}, 8 \mathrm{~Hz}), 6.26$ (d, 2H, $8 \mathrm{~Hz}), 5.12(\mathrm{~d}, 2 \mathrm{H}, 2 \mathrm{~Hz}), 4.65$ (d, 2H, $2 \mathrm{~Hz}), 2.46-2.01(\mathrm{~m}, 8 \mathrm{H}), 1.79(\mathrm{~s}, 6 \mathrm{H})$, $1.17(\mathrm{t}, 6 \mathrm{H}, 8 \mathrm{~Hz}), 0.88(\mathrm{t}, 6 \mathrm{H}, 8 \mathrm{~Hz})$.

Variable Temperature ${ }^{1} \mathbf{H}$ NMR Studies of 3 . In an inert atmosphere drybox, an NMR tube was charged with $13 \mathrm{mg}$ of $\mathbf{3}$ and $0.6 \mathrm{ml} \mathrm{C}_{6} \mathrm{D}_{6}$. The spectrometer was allowed to equilibrate for 10 minutes at each measurement temperature prior to collection of the ${ }^{1} \mathrm{H}$ NMR spectrum. The integration of the peaks representative of the $-\mathrm{OCH}_{2} \mathrm{O}$ - region for 3a and $\mathbf{3 b}$ were used to calculate the equilibrium constant $\mathrm{K}_{\mathrm{eq}}$ according to the equation $\mathrm{K}_{\mathrm{eq}}=[\mathbf{3 a}] /[\mathbf{3 b}]$, giving the following values:

$\begin{array}{cc}\mathrm{I}(\mathrm{K}) & \mathrm{K}(\mathrm{eq}) \\ 253.15 & 1.26 \\ 273.15 & 1.55 \\ 283.15 & 1.92 \\ 292.75 & 1.97 \\ 303.15 & 2.13 \\ 313.15 & 2.36 \\ 333.15 & 2.64\end{array}$

Crystallography. A summary of crystal data and collection parameters for the crystal structure of $\mathbf{4}$ are given in Table 1. Details of data collection and solution are given below. ORTEP diagrams were created using the ORTEP-3 software package. ${ }^{2}$

X-ray quality crystals were grown from a saturated ether solution that was cooled to $-25{ }^{\circ} \mathrm{C}$. A yellow crystalline block having dimensions of $0.29 \times 0.17 \times 0.12$ 
$\mathrm{mm}$ was mounted on a glass fiber using Paratone-N hydrocarbon oil. The crystal was transferred to a Siemens $\mathrm{SMART}^{3}$ diffractometer with a CCD area detector, centered in the $\mathrm{X}$-ray beam, and cooled to $-138{ }^{\circ} \mathrm{C}$ using a nitrogen-flow low-temperature apparatus that had been previously calibrated by a thermocouple placed at the same position as the crystal. An arbitrary hemisphere of data was collected using $0.3^{\circ} \omega$-scans that were counted for a total of $60 \mathrm{~s}$ per frame. The data were integrated by the program SAINT ${ }^{4}$ to a maximum $2 \theta$ value of $47.2^{\circ}$, and the final unit cell parameters were determined by least-squares analysis of 5204 reflections with $\mathrm{I}>10 \sigma(\mathrm{I})$. Data analysis using Siemens XPREP $^{5}$ gave a primitive triclinic cell and based on the successful solution and refinement of the structure, the space group was determined to be $P-1$ (\#2). An empirical absorption correction was applied using SADABS. ${ }^{6}$

Of the 37986 reflections that comprised the hemisphere, 18213 were unique $\left(\mathrm{R}_{\text {int }}\right.$ $=0.047)$, and the equivalent reflections were averaged. The structure was solved by direct methods using the Shelxtl ${ }^{7}$ software package. The final model included four ethyl groups that displayed methyl carbons disordered over two positions each. Additionally, there was one-half an equivalent of diffuse disordered diethyl ether. All non-hydrogen atoms of the zirconium complex were refined anisotropically, while the disordered diethyl ether was refined isotropically. Hydrogen atoms were included but not refined for all ordered atoms. The final cycle of full-matrix least squares refinement was based on 18213 observed reflections and 552 variable parameters and converged yielding final residuals: $\mathrm{R}=0.0684, \mathrm{R}_{\mathrm{all}}=0.1275$, and $\mathrm{GOF}=1.042$. 
Table 1: Crystal Data and Collection Parameters of $\mathrm{L}_{2} \mathrm{Zr}\left(\mathrm{CH}_{2} \mathrm{SiMe}_{3}\right)_{2}$ (4)

\begin{tabular}{|c|c|}
\hline Compound & 4 \\
\hline Formula & $\mathrm{C}_{46} \mathrm{H}_{62} \mathrm{~N}_{2} \mathrm{O}_{4} \mathrm{Si} \mathrm{Z}_{2} \mathrm{Zr} \cdot 1 / 2 \mathrm{C}_{4} \mathrm{H}_{10} \mathrm{O}$ \\
\hline Formula weight & 891.47 \\
\hline Space group & $P-1(\# 2)$ \\
\hline Temperature $\left({ }^{\circ} \mathrm{C}\right)$ & -138 \\
\hline$a(\AA)$ & $11.4685(6)$ \\
\hline$b(\AA)$ & $12.6056(6)$ \\
\hline$c(\AA)$ & $18.3425(9)$ \\
\hline$\alpha\left({ }^{\circ}\right)$ & $81.983(1)$ \\
\hline$\beta\left({ }^{\circ}\right)$ & $77.081(1)$ \\
\hline$\gamma\left({ }^{\circ}\right)$ & $65.461(1)$ \\
\hline$V\left(\AA^{3}\right)$ & $2347.9(2)$ \\
\hline$Z$ & 2 \\
\hline $\operatorname{Density}_{\text {calc }}\left(\mathrm{g} / \mathrm{cm}^{3}\right)$ & 1.261 \\
\hline Diffractometer & Siemens SMART \\
\hline Radiation & $\operatorname{Mo}^{-K_{\alpha}}(\lambda=0.71069 \AA)$ \\
\hline Monochromator & Graphite \\
\hline Detector & $\mathrm{CCD}$ area detector \\
\hline Scan type, width & $\omega, 0.3^{\circ}$ \\
\hline Scan speed & $60 \mathrm{~s} /$ frame \\
\hline Reflections measured & Hemisphere \\
\hline $2 \theta$ range $\left({ }^{\circ}\right)$ & $3-47.2$ \\
\hline Crystal dimensions (mm) & $0.29 \times 0.17 \times 0.12$ \\
\hline Reflections measured & 37986 \\
\hline Unique reflections & 18213 \\
\hline Observations & 18213 \\
\hline Parameters & 552 \\
\hline $\mathrm{R}, \mathrm{R}_{\mathrm{w}}, \mathrm{R}_{\mathrm{all}}$ & $0.0684 ; 0.1275 ; 0.1987$ \\
\hline GOF & 1.042 \\
\hline
\end{tabular}




\section{References}

(1) Blake, A. J.; Collier, P. E.; Dunn, S. C.; Li, W. S.; Mountford, P.; Shishkin, O. V. J. Chem. Soc., Dalton Trans. 1997, 1549.

(2) Farrugia, L. J. J. Appl. Cryst. 1997, 30, 565.

(3) SMART: Area-Detector Software Package, v5.625; Bruker AXS, Inc.: Madison, WI, 1997-2001.

(4) SAINT: SAX Area-Detector Integration Program, V6.22; Bruker AXS, Inc.: Madison, WI, 1997-2001.

(5) XPREP: Reciprocal Space Exploration Program, V6.12; Bruker AXS, Inc.: Madison, WI, 2001.

(6) SADABS: Bruker/Siemens Area Detector Absorption Program, V2.03; Bruker AXS, Inc.: Madison, WI, 2001.

(7) SHELXL-97: Structure Solution Program, V6.10; Bruker AXS, Inc.: Madison, WI, 2000. 